\title{
Stress responses upon starvation and exposure to bacteria in the ant Formica exsecta
}

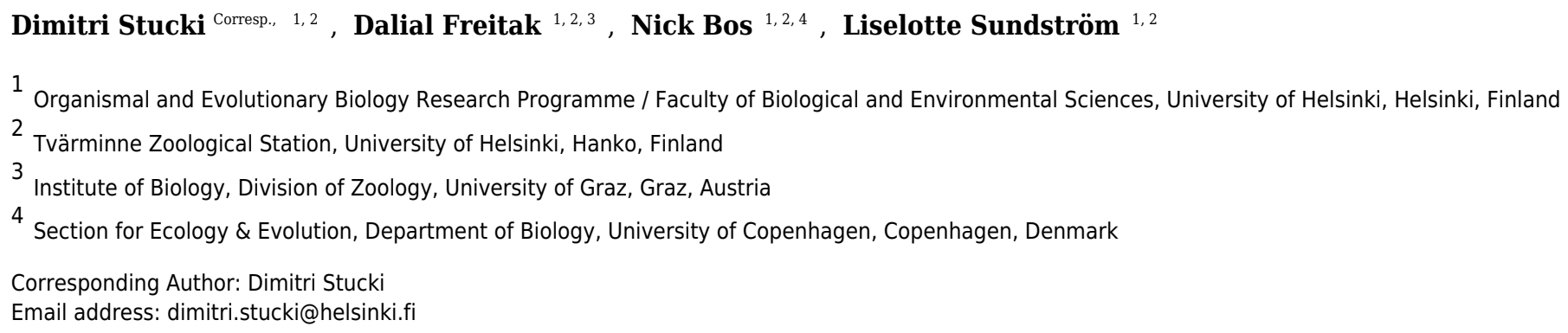

Organisms are simultaneously exposed to multiple stresses, which requires regulation of the resistance to each stress. Starvation is one of the most severe stresses organisms encounter, yet nutritional state is also one of the most crucial conditions on which other stress resistances depend. Concomitantly, organisms often deploy lower immune defenses when deprived of resources. This indicates that the investment into starvation resistance and immune defenses is likely to be subject to trade-offs. Here we investigated the impact of starvation and oral exposure to bacteria on survival and gene expression in the ant Formica exsecta. Of the three bacteria used in this study, only S. marcescens increased the mortality of the ants, whereas exposure to E. coli and P. entomophila alleviated the effects of starvation. Both exposure to bacteria and starvation induced changes in gene expression, but in different directions depending on the species of bacteria used, as well as on the nutritional state of the ants. 
1 Stress responses upon starvation and exposure to bacteria in the ant Formica exsecta

2

3 Authors:

4 Dimitri Stucki ${ }^{1,2}$ : dimitri.stucki@helsinki.fi $*$ Corresponding author

$5 \quad$ Dalial Freitak ${ }^{1,2,3}$ : dalial.freitak@uni-graz.at

6 Nick Bos ${ }^{1,2,4}$. nick.bos@,helsinki.fi

7 Liselotte Sundström ${ }^{1,2}$ : liselotte.sundstrom@helsinki.fi

8 1) Organismal and Evolutionary Biology Research Programme, Faculty of Biological and

9 Environmental Sciences, University of Helsinki, PO Box 65 (Viikinkaari 1), FI-00014 Helsinki,

10 Finland

11 2) Tvärminne Zoological Station, University of Helsinki, FI-10900 Hanko, Finland

12 3) Institute of Biology, Division of Zoology, University of Graz, Universitätsplatz 2, 8010 Graz, 13 Austria

14 4) Section for Ecology \& Evolution, Department of Biology, University of Copenhagen, 2100

15 Copenhagen, Denmark 
16 Abstract

17 Organisms are simultaneously exposed to multiple stresses, which requires regulation of the

18 resistance to each stress. Starvation is one of the most severe stresses organisms encounter, yet

19 nutritional state is also one of the most crucial conditions on which other stress resistances

20 depend. Concomitantly, organisms often deploy lower immune defenses when deprived of

21 resources. This indicates that the investment into starvation resistance and immune defenses is

22 likely to be subject to trade-offs. Here we investigated the impact of starvation and oral exposure

23 to bacteria on survival and gene expression in the ant Formica exsecta. Of the three bacteria used

24 in this study, only $S$. marcescens increased the mortality of the ants, whereas exposure to E. coli

25 and $P$. entomophila alleviated the effects of starvation. Both exposure to bacteria and starvation

26 induced changes in gene expression, but in different directions depending on the species of

27 bacteria used, as well as on the nutritional state of the ants.

31 Background

32 Organisms are at all times exposed to a wide variety of stresses, necessitating careful regulation

33 of responses to each stress (Mooney, Winner \& Pell, 1991; Broom \& Johnson, 1993). Ultimately

34 this generates trade-offs between different stress responses (Harshman, Hoffmann \& Clark,

35 1999; Hoffmann et al., 2005; Marshall \& Sinclair, 2010). As a consequence, natural selection on

36 stress resistance often affects multiple traits (Chapin III, Autumn \& Pugnaire, 1993; Vinebrooke 
37 et al., 2004), which may lead to different evolutionary trajectories, depending on the stresses an

38 organism encounters. Thus, in order to understand the eco-evolutionary mechanisms of stress

39 resistance, it is important to understand how organisms manage their response to multiple

40 stresses.

42 One of the most crucial and frequent stresses that organisms encounter is food deprivation.

43 Consequently, organisms have evolved ways to prepare for unfavorable periods with reduced

44 access to resources. For insects, the most common ways of accumulating energy reserves is via

45 the glycogen and triglycerides reserves in the fat body (Chapman, 2012), from where they can be

46 mobilized when needed. Glycolipoproteins, such as Arylphorin and Vitellogenin serve to store

47 resources in the hemolymph, but they also transport lipids from the fat body to organs.

48 Therefore, in times of resource limitation, an increased production of such proteins may increase

49 the release of stored resources. For energy management, insects also rely on the insulin pathway,

50 which is involved in the release of stored resources (Wu \& Brown, 2006). Thus, increased

51 expression of genes associated with the insulin pathway could improve resistance to starvation.

52 However, resistance to starvation can also be gained by lowering metabolic activity to reduce

53 energy consumption (Rion \& Kawecki, 2007). Furthermore, the regulation of genes involved in

54 adaptation to resource limitation likely depends on further factors, such as the presence of

55 parasites and the potential immune responses.

57 Given that host immune responses are often costly to maintain under food limitation

58 (Kraaijeveld, Ferrari \& Godfray, 2002; McKean et al., 2008), responses to starvation and 
59 immune defenses are likely constrained by trade-offs. Immune defenses not only incur a

60 metabolic cost, but are often also damaging to the host organism, due to immunopathology (Sadd

$61 \&$ Siva-Jothy, 2006). Therefore, selection is likely to favor reduced expression of immunity in

62 periods of low parasite pressure and/or low food availability. Such stress-dependent

63 immunosuppression could render food-deprived individuals prone to infections by opportunistic

64 pathogens (Dedet \& Pratlong, 2000), and create a trade-off between immune defenses and

65 starvation resistance. In many insects starvation has been associated with reduced immune

66 responses (Furlong \& Groden, 2003; Yang, Ruuhola \& Rantala, 2007), and, conversely, parasite

67 infection has been shown to reduce starvation resistance in the host organism (Hoang, 2001).

68 This suggests that the nutritional status influences both the expression of immune genes, and the

69 survival of infections.

71 Here we investigated a potential trade-off in starvation resistance and immune defenses against

72 bacteria in a natural population of the ant Formica exsecta. We expected to find increased mortality of the ants upon exposure to bacteria, which we assumed to become more pronounced

74 after the ants were starved, as a manifestation of the expected trade-off between starvation resistance and immune defenses. The aim of the study was to investigate how this trade-off is reflected in the expression of selected genes linked to starvation resistance and immune defenses.

77 The genes investigated here included potential starvation resistance genes, as well as a set of

78 immune defense genes. We compared worker ants on their ability to withstand food deprivation, 79 and to resist oral exposure to the entomopathogenic gram-negative bacteria Serratia marcescens 80 and Pseudomonas entomophila, as well as the response in gene expression upon these stresses.

81 These two entomopathogenic bacteria, S. marcescens (DB10) and P. entomophila (L48), were 
82 originally isolated from Drosophila melanogaster, and were shown to infect insects through oral

83 ingestion (Flyg, Kenne \& Boman, 1980; Dillon et al., 2005; Vodovar et al., 2005, 2006). Both

84 bacteria are soil dwelling opportunistc pathogens, with a wide range of potential hosts (Grimont

85 \& Grimont, 1978; Vallet-Gely et al., 2010). In addition, as a non-pathogenic sham-control we

86 used the benign gut-bacterium Escherichia coli (K12), originally isolated from a human stool

87 sample (Bachmann, 1972), and frequently used as model organism in insect infection assays

88 (Bartholomay et al., 2004; Altincicek et al., 2011; Freitak et al., 2014; Mikonranta et al., 2014).

Material \& Methods

91 Animals

92 For this study we used a population of the ant Formica exsecta located in southwestern Finland

93 in the municipality of Raseborg, Prästkulla (FI) (59 $\left.58^{\prime} 44.6^{\prime \prime} \mathrm{N} 23^{\circ} 20^{\prime} 50.9^{\prime \prime} E\right)$. Our sampling

94 procedures are non-destructive and do not cause harm to the field colonies. No federal laws

95 apply to the collection and scientific study of insects in Finland, and the study-site at Prästkulla

96 is not under protection. The specific population consists of nests with multiple reproductive

97 queens (polygyne), hence the relatedness, and consequently the genetic similarity, among

98 workers is low, yet genetic differences between individual nests is not expected to be high

99 (Sundström, Seppä \& Pamilo, 2005). In August 2013, we collected adult workers and nest

100 material from inside nine nest mounds, and transferred the workers, and ca. $5 \mathrm{dl}$ of nest material

101 immediately into nest boxes $(30 \mathrm{~cm}$ x $20 \mathrm{~cm} \times 15 \mathrm{~cm})$, lined with Fluon ${ }^{\circledR}$ (Whitford) in order to

102 prevent the ants from escaping. The ants were allowed to acclimatize to the laboratory conditions

103 for 24 hours at ambient room temperature $\left(23^{\circ} \mathrm{C}\right)$, and were fed daily ad libitum with a 
104 standardized diet based on agar, honey and egg (Bhatkar \& Whitcomb, 1970).

105

106 Experimental design

107 To start the experiment we transferred 20 monomorphic worker ants from each nest into eight

108 Fluon ${ }^{\circledR}$ coated pots $(\varnothing 7 \mathrm{~cm}, \mathrm{~h}: 5 \mathrm{~cm})$ with a plaster bottom (eight pots per nest). To maintain

109 humidity, an open $1.5 \mathrm{ml}$ tube filled with water, and a piece of cotton was placed in each pot, so

110 that the relative humidity was maintained at ca. $75 \%$. The ants were also allowed to acclimatize

111 to the experimental pots for 24 hours before the start of the experiment, and were fed with

$112 \sim 100 \mu 1$ of the standard diet. After 24 hours of acclimatization dead ants were replaced from the

113 main colony nest boxes to maintain a starting group size of 20 ants per treatment.

115 At the start of the experiment the eight pots per colony (72 pots, 20 ants each) were divided into

116 four groups that corresponded to four Exposure treatments (18 pots per Exposure) including a

117 control (factor denoted as Exposure). For these four Exposures we supplemented $\sim 100 \mu 1$

118 standard diet either with $50 \mu 1$ of clear LB-medium (Exposure denoted as control-exposure), or

119 with $50 \mu 1$ of concentrated bacteria growth culture (Exposures: $S$. marcescens, E. coli, and $P$.

120 entomophila), leading to a final volume of $150 \mu \mathrm{l}$ of food, which was provided in a detached cap

121 of a plastic test tube. Each day, a new cap was used and the old food was removed from the pots.

122 In order to provide a standardized amount of live bacteria each day, the growth-cultures were

123 renewed daily by inoculation with $30 \mu \mathrm{l}$ of overnight culture in $10 \mathrm{ml} \mathrm{LB}$-medium at $37^{\circ} \mathrm{C}$

124 without shaking. We concentrated the bacterial culture by spinning the overnight culture tubes

125 for $3 \mathrm{~min}$ at $8,000 \mathrm{rpm}$, removed all liquid LB-medium and subsequently suspended the remaining 
126 pellet in $1 \mathrm{ml}$ clear LB-medium, which led to a final concentration of $\mathrm{ca} .10^{8} \mathrm{cfu} / \mathrm{ml}$ for each

127 Exposure.

128

129 The experiment featured two phases. For the first seven days (first phase), the ants in both pots

130 of each Exposure (18 pots per Exposure) were fed daily $\sim 150 \mu 1$ diet according to the assigned

131 Exposure. After seven days, the second phase started (factor denoted as Treatment), during

132 which the ants in one of the two pots of each Exposure (9 pots per Exposure and Treatment)

133 were deprived of food for the next seven days (denoted as starved), whereas those in the other

134 pot continued to receive food on their assigned Exposure (denoted as continuously exposed).

135 This design allowed us to assess the combined effects of oral bacterial exposure and starvation,

136 as the effect of starvation could have been negated, if oral exposure had followed the starvation 137 step.

139 Throughout the experiment mortality was recorded daily, and dead ants were removed from the

140 pots. To assess the change in gene expression induced by the treatments, we randomly selected

141 three ants per pot for qPCR analysis on day nine after the beginning of the experiment (i.e. two

142 days after the onset of the second phase). Pilot experiments suggested that starvation-induced

143 mortality set in around two days after removal of the food, as we observed a 30\% drop in

144 survival within the first two days, which then petered out during the following days. Thus, we

145 expected effects on gene expression to be pronounced at this time point. Each colony constituted

146 one biological replicate per Exposure/Treatment combination. For each pot, three ants were

147 placed in $300 \mu 1$ Isol-RNA Lysis Reagent (5 Prime), and cut into small pieces. The samples were 
148 then stored at $-80^{\circ} \mathrm{C}$ until further processing.

149

150 Gene expression analysis

151 To extract the RNA we homogenized the thawed samples in $600 \mu 1$ Isol-RNA Lysis Reagent (5

152 Prime) with two stainless steel beads using a TissueLyser (Qiagen). We then added 400 $\mu 1$ Isol-

153 RNA Lysis Reagent and $150 \mu 1$ chloroform (Sigma). After mixing we centrifuged the samples for

$15410 \mathrm{~min}$ at $13,000 \mathrm{rpm}$ at $4^{\circ} \mathrm{C}$, and transferred the upper, transparent phase, containing the RNA, to

155 a new, autoclaved $1.5 \mathrm{ml}$ tube, and supplemented with $500 \mu \mathrm{l}$ isopropanol (Sigma, min. 99\%).

156 After mixing, we let the suspended RNA precipitate over night at $-20^{\circ} \mathrm{C}$, and then centrifuged the

157 samples for $30 \mathrm{~min}$ at $13,000 \mathrm{rpm}$ at $4^{\circ} \mathrm{C}$ to sediment the RNA. After removal of the supernatant,

158 we washed the pellet on ice with $500 \mu 180 \% \mathrm{EtOH}$ (Altia Oyj), and centrifuged for 10min at

$15913,000 \mathrm{rpm}$ at $4^{\circ} \mathrm{C}$. After drying the pellet we dissolved the RNA in autoclaved $\mathrm{ddH}_{2} \mathrm{O}$. We

160 measured the concentration and quality of the RNA photospectrometrically with a NanoDrop

161 (PEQ-Lab), and eliminated possible gDNA contamination by DNase digest (DNase I, RNase-

162 free; ThermoScientific) before cDNA synthesis with a blend of oligo(dT) and random primers

163 (iScript cDNA Synthesis Kit; Bio-Rad). For cDNA synthesis we used 1 $\mu$ g RNA for each sample,

164 and afterwards diluted the resulting $20 \mu \mathrm{l}$ cDNA in $80 \mu \mathrm{l}$ autoclaved $\mathrm{ddH}_{2} \mathrm{O}$.

165

166 As target genes we chose eight genes representing immune defense mechanisms on a broad

167 scale. We included two storage protein coding genes Vitellogenin 1 (Vg1) and Arylphorin

168 (Aryl), both of which also have immunological functions (Amdam et al., 2004; Zhu et al., 2009),

169 one Insulin Receptor (IR3), two antimicrobial peptides Lipopolysaccharide-binding protein 
170 (LPS-bp) and Hymenoptaecin (Hyme), the two cascade molecules Pro-Phenoloxidase (PPO) and

171 Toll-receptor (Toll), as well as the enzyme Lysozyme C (LysC), which has a double role in

172 digestion and immune defense (Cançado et al., 2007; Kajla et al., 2010). A list of the primer

173 sequences is provided in the supplementary Table S1.

174

175 We designed qRT-PCR primers using the online Primer3 internet-based interface

176 (http://www.ncbi.nlm.nih.gov/tools/primer-blast/) (Untergasser et al., 2012). Primers were

177 designed by the rules of highest maximum efficiency and sensitivity (Table S1), to avoid

178 formation of self- and hetero-dimers, hairpins, and self-complementarity. Gene-specific primers

179 were designed on the basis of the sequences obtained from $F$. exsecta transcriptome (Johansson 180 et al., 2013). Q-RT-PCR was performed on 384-well plates on a CFX384 Touch ${ }^{\mathrm{TM}}$ Real Time

181 PCR Detection System (Biorad) using iQ ${ }^{\mathrm{TM}}$ SYBR ${ }^{\circledR}$ Green Supermix (Bio-Rad), with an

182 initiation of $3 \mathrm{~min}$ at $95^{\circ} \mathrm{C}, 40$ cycles of $15 \mathrm{sec}$ at $95^{\circ} \mathrm{C}$ for denaturation, followed by $45 \mathrm{sec}$ at

$18358^{\circ} \mathrm{C}$ for annealing/extension, and a final step for $7 \mathrm{~min}$ at $95^{\circ} \mathrm{C}$. All the Q-RT-PCR assays were

184 run using two technical replicates, which were assessed for consistency and outliers (one case),

185 and subsequently averaged before normalization. Non-detects (no amplification signal within 40

186 qPCR cycles) were set to the maximal cycle number (i.e. $\mathrm{Ct}=40)$, or removed in case the second

187 technical replicate showed amplification.

188

189 Statistical analysis

190 We tested for the effect of ingestion of bacteria (Exposure), and subsequent starvation

191 (Treatment) on the survival of the ants by using mixed effects Cox proportional-hazard 
192 regression with Exposure (control-exposure, E. coli, P. entomophila, S. marcescens) as fixed

193 factor, Treatment (Continuously exposed, Starved) as time-dependent factor. The time-dependent

194 factor captures the fact that the ants of both Treatments were essentially treated the same during

195 the first phase of the experiment. Furthermore we included the interaction between the two

196 factors. To account for differences among the colonies, we used colony as random intercept. As

197 each colony was exposed to each Treatment and Exposure only once, there is only one unique

198 combination of colony, Treatment and Exposure, which corresponds to the pot. As each pot

199 reflected the colony, specifying pot as a random slope would allow for different reaction norms

200 of the colonies to each treatment. Unfortunately, random slopes are not yet available in the

201 survival packages for R. A random intercept term is not appropriate, as it would fix the same

202 slope for all pots (i.e. colonies), and further reduce the power of the analysis, potentially leading

203 to the acceptance of a false null hypothesis. We therefore decided to omit pot as a random factor.

204 In case of a significant interaction between Treatment and Exposure, we examined pairwise

205 mortality risks as post-hoc analysis, correcting for multiple comparisons using false discovery

206 rates (Benjamini \& Hochberg, 1995). Due to the limited sample size we focused the statistical

207 inference on a set of planned comparisons: i) between starved and continuously exposed

208 individuals within each Exposure and ii) between each Exposure and the control exposure within

209 each Treatment. We used a single model including all Exposures to allow the reader the

210 comparison among all Exposures, just without the aid of statistical inference. We tested for

211 overall effects of Treatment, Exposure and the interaction through likelihood ratio tests. We

212 compared the full model against an intercept only model, and against a model with dropped

213 interaction term. 
215 Gene expression was analyzed on the level of normalized Ct values. Three ants were pooled

216 from each pot, which led to a final sample size of nine samples per Treatment and Exposure. To

217 test for differences in gene expression across Exposures and Treatments, we first performed

218 Principal Component Analysis (PCA) on the inverted normalized Ct-values. We inverted the Ct-

219 values because they are negatively correlated with the transcript level (i.e. higher Ct-values

220 indicate lower gene expression levels). Normalization of the Ct-values was done with the

221 NORMA-gene algorithm, which does not require reference genes (Heckmann et al., 2011). We

222 assessed the data for suitability for a PCA: The Kaiser-Meyer-Olkin (KMO) measures of the

223 eight genes ranged between 0.61 and 0.84 , and thus indicated a sampling adequacy acceptable

224 for PCA (Field, Miles \& Field, 2012). A Bartlett's test of sphericity also indicated sufficiently

225 strong correlations between the genes $\left(\mathrm{X}^{2}=321.4, \mathrm{p}<0.0001\right)$, and the determinant of the

226 correlation matrix $(\mathrm{det}=0.009)$ indicated that the correlations were not too strong for a PCA (i.e.

227 det $>0.00001$ (Field, Miles \& Field, 2012)).

228 We used an unrotated PCA for component selection, and consulted the scree plot of the

229 Eigenvalues for the relative weights of the components. Two components had an Eigenvalue $>$

2301.0 (Table S2), but the scree plot showed a high relative weight only for PC1, whereas the weight

231 of PC2 was not distinctively higher compared to the subsequent components (Figure S1). A

232 preliminary analysis including PC1 and PC2 showed no significant effects of PC2 for any of the

233 factors (Supplementary Tables S3 \& S4). We therefore decided to use only PC1 for the analysis.

234 The PC scores were then used as dependent variables in a linear mixed effects model to test for

235 an association of PC1 with Exposure and Treatment. Colony was added as a random intercept. In

236 case of a significant interaction of Treatment and Exposure, we examined pairwise gene

237 expression differences as post-hoc analysis, correcting for multiple comparisons using false 
238 discovery rates. We used the same procedure for post-hoc comparisons as for the survival data.

239 To assess the contribution of each gene to the effects found for PC1, we here arbitrarily define

240 the loadings as strong (loading $0.67-1.0$ ), moderate (loading $0.33-0.66)$, or weak $(0.0-0.32)$.

241 Because the PCA indicated multicollinearity among the genes, we did not use a gene-by-gene

242 analysis as a basis for our conclusions, but provide one as supplementary material (Figure S2,

243 Table S5).

245 We assessed the validity for a cox-proportional hazard model with a proportional hazard test

246 (Grambsch \& Therneau, 1994), which did not reveal any indication for non-proportional hazards

247 (Chi-squared: 9.17, $\mathrm{p}=0.24$ ). As a method to test for the presence of proportional hazards is not

248 available for mixed effects Cox-proportional hazard models, we specified a default cox-ph

249 model, with Colony specified as frailty term (Therneau, Grambsch \& Pankratz, 2003). The

250 validity of the linear mixed effects models was assessed visually through inspection of the

251 residuals and the leverage, which showed no evidence for misspecification of the model. For all

252 statistical tests we used a threshold level of significance of $\alpha=0.05$. Statistical analysis was

253 carried out in R version 3.0.2 (R Core Team, 2015), using the packages survival (Therneau,

254 2013), coxme (Therneau, 2018), psych (Revelle, 2016), lme4 (Bates et al., 2014), lmerTest

255 (Kuznetsova et al., 2014), and multcomp (Hothorn, Bretz \& Westfall, 2008).

256

257 Results

258 Survival 
259 Overall the Cox-proportional hazard regression revealed significant effects of the treatments on

260 the survival of the ants (Likelihood ratio test: Chi-square $=476.89, \mathrm{df}=7, \mathrm{p}<0.0001$ ). The

261 interaction was close to significant (Likelihood ratio test: Chi-square $=7.41, \mathrm{df}=3, \mathrm{p}=0.06$ ). Given

262 the borderline effect, we decided to retain the interaction term in the model as the log likelihood

263 was slightly higher when the interaction term was included $\left(\operatorname{loglik} \mathrm{Full}_{\mathrm{F}}=-4032.0, \log \operatorname{lik}_{\text {Reduced }}=-\right.$ 264 4035.7).

265 Starvation reduced the survival of the ants in all exposure regimes (Figure 1, Tables $1 \& 2$ ).

266 Exposure to $S$. marcescens significantly reduced survival compared to the respective controls,

267 both under continuous feeding and starvation (Figure 1a, comparisons between black and red

268 continuous lines, and dotted lines, respectively, Tables 1 \& 2). No significant changes in

269 mortality emerged when the ants were continuously fed and exposed to E. coli or P. entomophila

270 (Figure $1 \mathrm{~b}$ and c, comparison between black and red continuous lines, Tables 1 \& 2). However,

271 when these ants were starved, they survived significantly longer if they had been exposed to $E$.

272 coli or P. entomophila, than if they had been exposed to the control-exposure (Figure $1 \mathrm{~b}$ and $\mathrm{c}$, 273 comparison between black and red hatched lines, Tables $1 \& 2$ ).

274

275 Gene expression

276 From the PCA on gene expression we retained one component, which explained $51 \%$ of the

277 variation (Table S2). The loadings of all genes were positive, and either moderate (Vg1: 0.60;

278 PPO: 0.66; Hyme: 0.36; LPS-bp: 0.62), or strong (Aryl: 0.76; IR3: 0.88; LysC: 0.82; Toll: 0.89).

279 This shows that all genes were regulated in the same direction and to a similar degree (apart from

280 Hymenoptaecin), and that the PC1 scores therefore reflect the general level of gene expression. 
281 Overall the mixed effects model revealed significant effects of the treatments on gene expression

282 (Likelihood ratio test: Chi-square $=177.2, \mathrm{df}=2, \mathrm{p}<0.0001$ ), with a significant interaction term

283 (Likelihood ratio test: Chi-square $=130.81, \mathrm{df}=3, \mathrm{p}<0.0001)$. Continuous exposure to $S$.

284 marcescens led to a decrease in gene expression levels, compared to the control-exposure,

285 whereas the opposite occurred when the ants were starved (Figure 2, comparison between black

286 symbols, and white symbols, respectively, Tables $3 \&$ 4). By contrast, ants that were

287 continuously exposed to E. coli or P. entomophila showed higher gene expression levels than

288 ants of the control-exposure (Figure 2, comparison between black symbols). When the ants had

289 been exposed to E. coli, this also occurred after starvation, but not when they had been exposed

290 to P. entomophila (Figure 2, comparison between white symbols, Tables 3 \& 4).

291 Ants that had received either the control-exposure or P. entomophila treatment showed lower

292 gene expression levels after starvation, than during continuous exposure (Figure 2, comparison

293 between black and white symbols within regime). By contrast, the ants that had been exposed to

294 S. marcescens, showed significantly higher gene expression levels in starved, than in

295 continuously exposed individuals. Only ants that had been exposed to E. coli showed no

296 difference in gene expression between starved, and continuously exposed individuals (Figure 2,

297 Tables 3 \& 4).

299 Discussion

300 In this study we investigated the responses of the ant F. exsecta to two stresses; oral exposure to

301 bacteria and subsequent starvation. As expected, starvation induced a strong decrease in survival,

302 but the responses in combination with oral exposure to bacteria differed depending on the 
303 bacteria. We also found that both starvation and exposure influenced gene expression differently

304 depending on the bacteria used for exposure, and the feeding regime. However, we found no

305 evidence for a gene-level trade-off between investment in immune defenses and starvation

306 resistance, as none of the genes showed differential expression patterns (based on the fact that all

307 genes loaded positively on PC1) which indicated that the regulation of gene expression was

308 highly consistent upon each treatment.

310 As expected both starvation and exposure to $S$. marcescens induced increased mortality under

311 both feeding regimes (continuous food vs. starvation during the second stage). As these results

312 were expected and the statistical support is strong, we believe this result to be unaffected by the

313 omission of pot as a random effect. We found no evidence for additive effects from exposure

314 combined with starvation. In contrast, neither E. coli nor P. entomophila induced severe

315 mortality in the ants. This may indicate that these bacteria are not infectious to $F$. exsecta, either

316 due to a lack of the necessary infective mechanisms, or due to an efficient immune response

317 from the ants. Yet, surprisingly, exposure to $P$. entomophila and E. coli alleviated the effects of

318 starvation on mortality, at least when administered orally. This stands in contrast to previous

319 findings in insects, which showed an increase in the susceptibility to infections under food-

320 deprived conditions (Feder et al., 1997; Vass \& Nappi, 1998; Kraaijeveld, Ferrari \& Godfray,

321 2002; McKean et al., 2008), or a decrease in starvation resistance when infected (Hoang, 2001).

322 Because the p-values of these results are relatively close to our alpha level of 0.05 it is possible

323 that this result may be biased due to the omission of pot as a random effect. Nevertheless, the

324 fact thet this effect was observed independently for two different bacteria, and that the parameter 325 estimate for $S$. marcescens points in the same direction, suggests that this is valid observation. 
327 A possible explanation for the reduced mortality during the starvation phase could be the

328 induction of anorexia (Adamo, 2005; Adamo, Fidler \& Forestell, 2007; Ayres \& Schneider,

329 2009), which in insects can either reduce or increase survival, depending on the pathogen (Ayres

330 \& Schneider, 2009). In such a scenario, infection leads to loss of appetite, and the ensuing

331 dietary restriction alters metabolic rates, which may increase starvation resistance (Chippindale

332 et al., 1993; Burger et al., 2007). Similarly, the ants may have sensed the food contamination and

333 reduced consumption,which may have primed their physiological resistance to starvation.

334 Reduced food consumption may also have led to the down-regulation of gene expression in ants

335 that were continuously exposed to $S$. marcescens. Thus, the reduced survival during continuous

336 exposure to $S$. marcescens may also reflect mortality from starvation. However, given that the

337 two other bacteria did not elicit such a response, the ants may not recognize all bacteria or the

338 response to contamination may depend on the bacteria. Finally, both bacteria could have added

339 nutritional value to the diet, which could alleviate the effects of starvation. At present these

340 explanations remain open to debate.

341

342 Starvation induced clear effects on gene expression in all treatments, but the patterns of change

343 differed considerably between exposure regimes. Gene expression increased significantly in

344 starved ants, compared to the control, when the ants had been exposed to E. coli or $S$.

345 marcescens before starvation, but not in those exposed to P. entomophila. Also in non-starved

346 ants the responses differed between the infection regimes, such that gene expression was up

347 regulated, compared to the control-exposure, in ants exposed to P. entomophila, and E. coli, but 
348 strongly down-regulated in those exposed to $S$. marcescens. The responses to P. entomophila,

349 and $E$. coli under non-starved conditions probably reflect a general immune response. The high

350 dose may have elicited a general immune response. Alternatively, this may be a prophylactic

351 response upon the detection of contaminated food. The response to $S$. marcescens may reflect a

352 trade-off between energy conservation and immune defense, or reflects an inability to uphold

353 immune defenses under severe infections. In the control-exposure, gene expression decreased

354 upon starvation, which likely reflects a mechanism to save resources under food limitation (Rion

$355 \&$ Kawecki, 2007). It is important to note, however, that the set of genes investigated here only

356 represent a subset of the genes involved in metabolic regulation and immune defenses.

357 Therefore, other genes involved in these regulation pathways may also have influenced the

358 effects of starvation. We discuss these results, and the comparisons within each exposure regime

359 in detail below.

361 When comparing starved and non-starved ants exposed to P. entomophila we found significantly

362 lower levels of gene expression in the starved group. This could indicate purging of infection, as

363 the ants at this point ceased to receive the supplemented food, and an activation of immune

364 responses therefore was not needed any more. This may also explain the enhanced survival of the

365 exposed ants, compared to the control, if the bacteria conveyed nutritional value. However, a

366 possible effect of additive nutritional value is speculative, and it remains to be tested whether

367 these bacteria provide a relevant quantity of nutritional value to insects. In the ants exposed to $E$.

368 coli an elevated level of gene expression was maintained also under starvation. This may indicate

369 that the naturally occurring E. coli was not directly targeted, but that the immune response was

370 maintained due to a higher sensitivity after exposure (Sadd \& Schmid-Hempel, 2006), or the 
371 presence of other pathogens. In this case the enhanced survival of exposed starved ants,

372 compared to the non-starved ones, remains unexplained, unless the bacteria conveyed nutritional

373 value also in this case. Unlike E. coli and P. entomophila, S. marcescens induced a down-

374 regulation of gene expression in non-starved ants, and an up-regulation in starved ones. As the

375 supply of $S$. marcescens also ceased upon the onset of starvation, the ants may have restored

376 gene expression levels despite starvation, and cleared the infection. This could explain the

377 absence of an additive negative effect of both challenges, infection and starvation.

378

379 In addition to behavioral and physiological adaptations against infection and starvation, social

380 insects have access to collective defenses (Cremer, Armitage \& Schmid-Hempel, 2007), which

381 might explain the absence of a trade-off between survival and immune defenses, both at the level

382 of individuals, and terms of gene expression. If the ants intensified their social defenses (e.g.

383 trophallactic prophylaxis (Hamilton, Lejeune \& Rosengaus, 2011)) when starved, the individual

384 defenses would have been less strained, and thus a trade-off between starvation resistance and

385 immune defenses would be less evident from the physiological response. Indeed, social

386 interactions were reported to increase when ants were starved for a longer period (Rueppell \&

387 Kirkman, 2005). If such interactions encompass social immune defenses, such as trophallactic

388 prophylaxis (Hamilton, Lejeune \& Rosengaus, 2011), this would indicate a synergistic interplay

389 of the defenses against starvation and infections.

391 The pattern of gene expression was very similar across all investigated genes and treatments

392 (Figure S2, Table S5). Furthermore, the observed patterns of gene expression were consistent 
393 among the colonies, which suggests that these stresses trigger conserved responses that are

394 common to the workers of $F$. exsecta. Given that the candidate genes represented a broad

395 selection of immune defense pathways, this suggests a systemic response to the stresses, and

396 shows the synergistic relationship between different immune pathways, such as the Toll and Imd

397 pathways (Tanji et al., 2007). It is, however, possible that the severity of the stresses prevented a

398 more nuanced response among genes, and instead induced a systemic response.

400 Conclusions

401 In conclusion our results indicate that exposure to some bacteria may have a beneficial effect on 402 starvation resistance in the ant $F$. exsecta. Although the underlying mechanism is not evident

403 from the regulation of the investigated genes, our results highlight the fact that the interplay

404 between nutritional state, and pathogen exposure is not regulated by straightforward trade-offs.

405 Instead the beneficial effect of exposure to bacteria on starvation resistance may reflect the

406 ubiquity of pathogens and resource scarcity in nature. Given that the responses to these stresses

407 were similar across all genes, as well as between colonies, this suggests that evolutionary

408 trajectories due to the presence of multiple stresses can result in conserved stress responses.

410 Acknowledgements

411 We thank our field assistants for their valuable help during sampling of the colonies, as well as

412 our suppliers of the bacteria strains, Lauri Mikonranta (University of Jyväskylä; S. marcescens \&

413 E. coli), and Bruno Lemaitre (University of Lausanne; P. entomophila). Furthermore we want to 
414 thank the editor Prof. Dr. Joseph Gillespie and two anonymous reviewers for valuable input on 415 the manuscript.

416

417

418 
419

420

421

422

423

424

425

426

427

428

429

430

431

432

433

434

435

436

437

438

439

440

441

442

443

444

445

446

447

448

449

450

References

Adamo SA. 2005. Parasitic suppression of feeding in the tobacco hornworm, Manduca sexta: parallels with feeding depression after an immune challenge. Archives of insect biochemistry and physiology 60:185-97. DOI: 10.1002/arch.20068.

Adamo SA., Fidler TL., Forestell CA. 2007. Illness-induced anorexia and its possible function in the caterpillar, Manduca sexta. Brain, Behavior, and Immunity 21:292-300. DOI: 10.1016/j.bbi.2006.10.006.

Altincicek B., Laughton AM., Udekwu KI., Gerardo NM. 2011. Escherichia coli K-12 pathogenicity in the pea aphid, Acyrthosiphon pisum, reveals reduced antibacterial defense in aphids. Developmental and Comparative Immunology 35:1091-1097. DOI: 10.1016/j.dci.2011.03.017.

Amdam GV., Simões ZLP., Hagen A., Norberg K., Schrøder K., Mikkelsen Ø., Kirkwood TBL., Omholt SW. 2004. Hormonal control of the yolk precursor vitellogenin regulates immune function and longevity in honeybees. Experimental Gerontology 39:767-773. DOI: 10.1016/j.exger.2004.02.010.

Ayres JS., Schneider DS. 2009. The Role of Anorexia in Resistance and Tolerance to Infections in Drosophila. PLoS Biology 7:e1000150. DOI: 10.1371/journal.pbio.1000150.

Bachmann BJ. 1972. Pedigrees of Some Mutant Strains of Escherichia coli K-12. Bacteriological Reviews 36:525-557.

Bartholomay LC., Cho W., Rocheleau TA., Boyle JP., Beck ET., Fuchs JF., Liss P., Rusch M., Butler KM., Wu RC., Lin S., Kuo H., Tsao I., Huang C., Liu T., Hsiao K., Tsai S., Yang U., Nappi AJ., Perna NT., Chen C., Christensen BM. 2004. Description of the Transcriptomes of Immune Response-Activated Hemocytes from the Mosquito Vectors Aedes aegypti and Armigeres subalbatus. Infection and Immunity 72:4114-4126. DOI: 10.1128/IAI.72.7.4114.

Bates D., Maechler M., Bolker BM., Walker S. 2014. lme4: Linear mixed-effects models using Eigen and S4.

Benjamini Y., Hochberg Y. 1995. Controlling the false discovery rate: a practical and powerful approach to multiple testing. Journal of the Royal Statistical Society. Series B 57:289-300.

Bhatkar A., Whitcomb WH. 1970. Artificial Diet for Rearing Various Species of Ants. Florida Entomologist 53:229-232. DOI: 10.2307/3493193.

Broom DM., Johnson KG. 1993. Stress and Animal Welfare. Dordrecht: Kluwer Academic 
Publishers.

452 Burger JMS., Hwangbo DS., Corby-Harris V., Promislow DEL. 2007. The functional costs and

453

454

455

456

457

458

459

460

461

462

463

464

465

466

467

468

469

470

471

472

473

474

475

476

477

478

479

480

481

482

483

484

benefits of dietary restriction in Drosophila. Aging Cell 6:63-71. DOI: 10.1111/j.14749726.2006.00261.x.

Cançado FC., Valério AA., Marana SR., Barbosa JARG. 2007. The crystal structure of a lysozyme c from housefly Musca domestica, the first structure of a digestive lysozyme. Journal of Structural Biology 160:83-92. DOI: 10.1016/j.jsb.2007.07.008.

Chapin III FS., Autumn K., Pugnaire F. 1993. Evolution of Suites of Traits in Response to Environmental Stress. The American Naturalist 142:S78-S92. DOI: 10.1086/285524.

Chapman RF. 2012. The Insects: Structure and Function. Cambridge: Cambridge University Press.

Chippindale AK., Leroi AM., Sung KB., Rose MR. 1993. Phenotypic plasticity and selection in Drosophila life-history evolution. I. Nutrition and the cost of reproduction. Journal of Evolutionary Biology 6:171-193. DOI: doi:10.1046/j.1420-9101.1993.6020171.x.

Cremer S., Armitage SAO., Schmid-Hempel P. 2007. Social immunity. Current biology 17:R693-R702. DOI: 10.1016/j.cub.2007.06.008.

Dedet J-P., Pratlong F. 2000. Leishmania, Trypanosoma and monoxenous trypanosomatids as emerging opportunistic agents. The Journal of eukaryotic microbiology 47:37-39. DOI: 10.1111/j.1550-7408.2000.tb00008.x.

Dillon RJ., Vennard CT., Buckling A., Charnley AK. 2005. Diversity of locust gut bacteria protects against pathogen invasion. Ecology Letters 8:1291-1298. DOI: 10.1111/j.14610248.2005.00828.x.

Feder D., Mello CB., Garcia ES., Azambuja P. 1997. Immune responses in Rhodnius prolixus: Influence of nutrition and ecdysone. Journal of Insect Physiology 43:513-519. DOI: 10.1016/S0022-1910(97)00010-3.

Field A., Miles J., Field Z. 2012. Discovering statistics Using R. London: SAGE Publications Ltd.

Flyg C., Kenne K., Boman HG. 1980. Insect Pathogenetic Properties of Serratia marcescens: Phage-resistant Mutants with a Decreased Resistance to Ceropia Immunity and a Decreased Virulence to Drosophila. Journal of General Microbiology 120:173-181. DOI: 10.1099/00221287-120-1-173.

Freitak D., Schmidtberg H., Dickel F., Lochnit G., Vogel H., Vilcinskas A., Freitak D., Schmidtberg H., Dickel F., Lochnit G., Vogel H., Vilcinskas A. 2014. The maternal transfer of bacteria can mediate trans-generational immune priming in insects. Virulence 5:547-554. 
485

486

487

488

489

490

491

492

493

494

495

496

497

498

499

500

501

502

503

504

505

506

507

508

509

510

511

512

513

514

515

516

517

518

DOI: $10.4161 /$ viru.28367.

Furlong MJ., Groden E. 2003. Starvation induced stress and the susceptibility of the Colorado potato beetle, Leptinotarsa decemlineata, to infection by Beauveria bassiana. Journal of Invertebrate Pathology 83:127-138. DOI: 10.1016/S0022-2011(03)00066-1.

Grambsch PM., Therneau TM. 1994. Proportional hazards tests and diagnostics based on weighted residuals. Biometrika 81:515-526. DOI: https://doi.org/10.1093/biomet/81.3.515.

Grimont PAD., Grimont F. 1978. The genus Serratia. Annual review of microbiology 32:221248. DOI: 10.1146/annurev.mi.32.100178.001253.

Hamilton C., Lejeune BT., Rosengaus RB. 2011. Trophallaxis and prophylaxis: social immunity in the carpenter ant Camponotus pennsylvanicus. Biology letters 7:89-92. DOI: 10.1098/rsbl.2010.0466.

Harshman LG., Hoffmann AA., Clark AG. 1999. Selection for starvation resistance in Drosophila melanogaster: Physiological correlates, enzyme activities and multiple stress responses. Journal of Evolutionary Biology 12:370-379. DOI: 10.1046/j.14209101.1999.00024.x.

Heckmann L-H., Sørensen PB., Krogh PH., Sørensen JG. 2011. NORMA-Gene: a simple and robust method for qPCR normalization based on target gene data. BMC bioinformatics 12:1-7. DOI: 10.1186/1471-2105-12-250.

Hoang A. 2001. Immune response to parasitism reduces resistance of Drosophila melanogaster to desiccation and starvation. Evolution 55:2353-2358. DOI: 10.1111/j.00143820.2001.tb00748.x.

Hoffmann AA., Hallas R., Anderson AR., Telonis-Scott M. 2005. Evidence for a robust sexspecific trade-off between cold resistance and starvation resistance in Drosophila melanogaster. Journal of Evolutionary Biology 18:804-810. DOI: 10.1111/j.14209101.2004.00871.x.

Hothorn T., Bretz F., Westfall P. 2008. Simultaneous Inference in General Parametric Models. Biometrical Journal 50:346-363. DOI: 10.1002/bimj.200810425.

Johansson H., Dhaygude K., Lindström S., Helanterä H., Sundström L., Trontti K. 2013. A metatranscriptomic approach to the identification of microbiota associated with the ant Formica exsecta. PLoS ONE 8:e79777. DOI: 10.1371/journal.pone.0079777.

Kajla MK., Andreeva O., Gilbreath TM., Paskewitz SM. 2010. Characterization of expression, activity and role in antibacterial immunity of Anopheles gambiae lysozyme c-1. Comparative Biochemistry and Physiology - B 155:201-209. DOI: 
519 Kraaijeveld AR., Ferrari J., Godfray HCJ. 2002. Costs of resistance in insect-parasite and insect520 parasitoid interactions. Parasitology 125:S71-S82. DOI: 10.1017/S0031182002001750.

521 Kuznetsova A., Brockhoff Bruun P., Bojesen H., Christensen R. 2014. lmerTest: Tests in Linear Mixed Effects Models.

Marshall KE., Sinclair BJ. 2010. Repeated stress exposure results in a survival-reproduction trade-off in Drosophila melanogaster. Proceedings of the Royal Society B: Biological Sciences 277:963-969. DOI: 10.1098/rspb.2009.1807.

McKean KA., Yourth CP., Lazzaro BP., Clark AG. 2008. The evolutionary costs of

527 immunological maintenance and deployment. BMC Evolutionary Biology 8:1-19. DOI:

528 10.1186/1471-2148-8-76.

Mikonranta L., Mappes J., Kaukoniitty M., Freitak D. 2014. Insect immunity : oral exposure to a bacterial pathogen elicits free radical response and protects from a recurring infection. Frontiers in Zoology 11:1-7.

Mooney HA., Winner WE., Pell EJ. 1991. Response of Plants to Multiple Stresses. San Diego: Academic Press, Inc.

R Core Team. 2015. R: A Language and Environment for Statistical Computing.

Revelle W. 2016. psych: Procedures for Psychological, Psychometric, and Personality Research.

Rion S., Kawecki TJ. 2007. Evolutionary biology of starvation resistance: What we have learned from Drosophila. Journal of Evolutionary Biology 20:1655-1664. DOI: 10.1111/j.14209101.2007.01405.x.

540

Rueppell O., Kirkman RW. 2005. Extraordinary starvation resistance in Temnothorax rugatulus (Hymenoptera, Formicidae) colonies: Demography and adaptive behavior. Insectes Sociaux 52:282-290. DOI: 10.1007/s00040-005-0804-2.

Sadd BM., Schmid-Hempel P. 2006. Insect immunity shows specificity in protection upon secondary pathogen exposure. Current biology 16:1206-1210. DOI:

Sadd BM., Siva-Jothy MT. 2006. Self-harm caused by an insect's innate immunity. Proceedings.

Sundström L., Seppä P., Pamilo P. 2005. Genetic population structure and dispersal patterns in Formica ants - a review. Annales Zoologici Fennici 42:163-177. DOI: 10.1007/BF00172934.

550 Tanji T., Hu X., Weber ANR., Ip YT. 2007. Toll and IMD Pathways Synergistically Activate an 551 Innate Immune Response in Drosophila melanogaster. Molecular and Cellular Biology 
553 Therneau TM. 2013. A Package for Survival Analysis in S.

554 Therneau TM. 2018. coxme: Mixed Effects Cox Models.

555 Therneau TM., Grambsch PM., Pankratz VS. 2003. Penalized Survival Models and Frailty.

556 Journal of Computational and Graphical Statistics 12:156-175. DOI:

$557 \quad 10.1198 / 1061860031365$.

558

559

Untergasser A., Cutcutache I., Koressaar T., Ye J., Faircloth BC., Remm M., Rozen SG. 2012.

560 Primer3-new capabilities and interfaces. Nucleic Acids Research 40:e115. DOI:

561

562 $10.1093 /$ nar/gks596.

563

Vallet-Gely I., Novikov A., Augusto L., Liehl P., Bolbach G., Péchy-Tarr M., Cosson P., Keel C., Caroff M., Lemaitre B. 2010. Association of Hemolytic Activity of Pseudomonas entomophila, a Versatile Soil Bacterium, with Cyclic Lipopeptide Production. Applied and Environmental Microbiology 76:910-921. DOI: 10.1128/AEM.02112-09.

Vass E., Nappi AJ. 1998. The effects of dietary yeast on the cellular immune response of Drosophila melanogaster against the larval parasitoid, Leptopilina boulardi. Journal of Parasitology 84:870-872.

Vinebrooke RD., Cottingham KL., Norberg J., Scheffer M., Dodson SI., Maberly SC., Sommer

570

571

572

573

574

575

576

577

578

579

580

581

582

583

584 U. 2004. Impacts of multiple stressors on biodiversity and ecosystem functioning: The role of species co-tolerance. Oikos 104:451-457. DOI: 10.1111/j.0030-1299.2004.13255.x.

Vodovar N., Vallenet D., Cruveiller S., Rouy Z., Barbe V., Acosta C., Cattolico L., Jubin C., Lajus A., Segurens B., Vacherie B., Wincker P., Weissenbach J., Lemaitre B., Médigue C., Boccard F. 2006. Complete genome sequence of the entomopathogenic and metabolically versatile soil bacterium Pseudomonas entomophila. Nature Biotechnology 24:673-679. DOI: $10.1038 / \mathrm{nbt} 1212$.

Vodovar N., Vinals M., Liehl P., Basset A., Degrouard J., Spellman P., Boccard F., Lemaitre B. 2005. Drosophila host defense after oral infection by an entomopathogenic Pseudomonas species. PNAS 102:11414-11419. DOI: 10.1073/pnas.0502240102.

Wu Q., Brown MR. 2006. Signaling and function of insulin-like peptides in insects. Annual Review of Entomology 51:1-24. DOI: 10.1146/annurev.ento.51.110104.151011.

Yang S., Ruuhola T., Rantala MJ. 2007. Impact of starvation on immune defense and other lifehistory traits of an outbreaking geometrid, Epirrita autumnata: a possible causal trigger for the crash phase of population cycle. Annales Zoologici Fennici 44:89-96. 
585 Zhu J-Y., Ye G-Y., Fang Q., Hu C., Akhtar Z-R. 2009. cDNA of an arylphorin-type storage 586 protein from Pieris rapae with parasitism inducible expression by the endoparasitoid wasp 587 Pteromalus puparum. Insect Science 16:227-236. DOI: 10.1111/j.1744-7917.2009.01252.x. 588

589 


\section{Figure $\mathbf{1}$ (on next page)}

Effect of treatments on ant surviva

Cumulative survival after oral exposure to bacteria and upon starvation. For each bacteria Exposure, the survival of the ants of the control Exposure is repeatedly shown in black for clarity, and the survival of the bacteria Exposure in red. The ants were either fed daily throughout the experiment (solid lines), or for a limited time followed by starvation (dashed lines). The vertical dashed line indicates the onset of starvation for the starvation treatment. Letters indicate significant differences within each Exposure and are not directly comparable between exposures. 


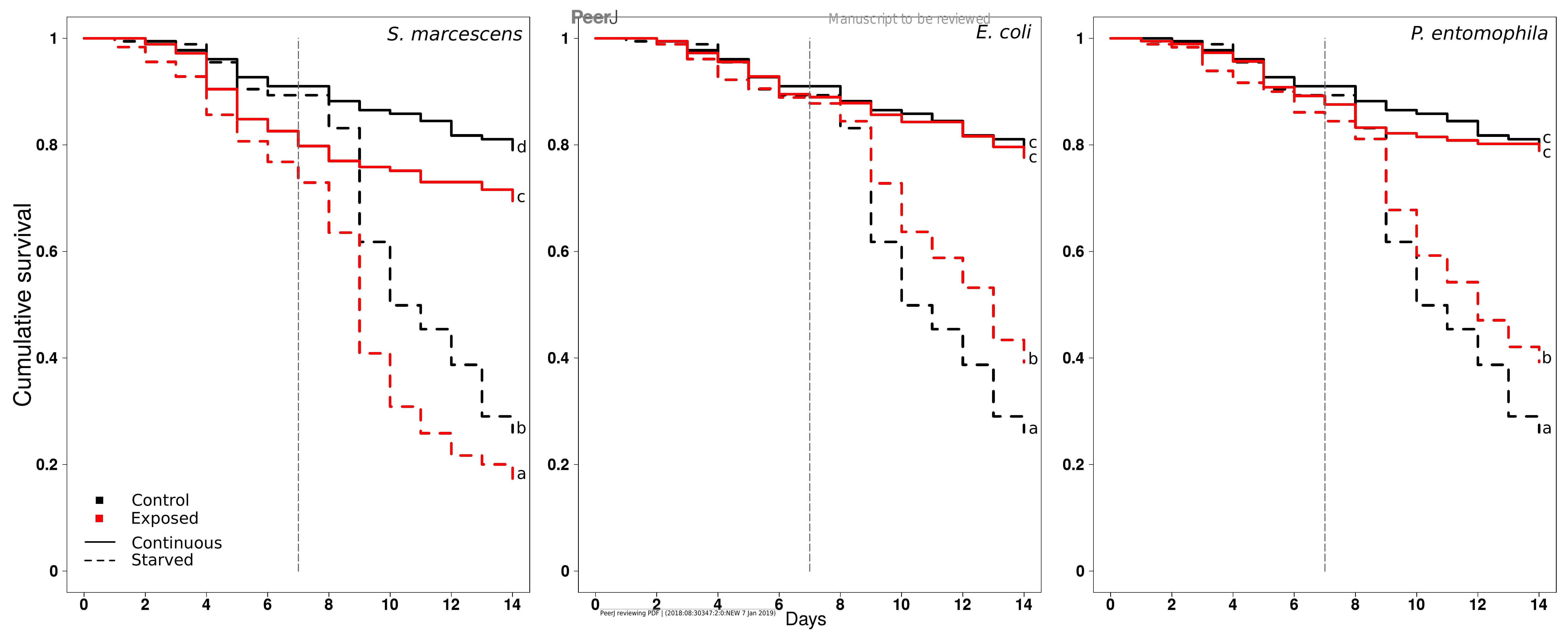




\section{Figure 2 (on next page)}

Effect of treatments on gene expression (PC1)

PC1-scores averaged across colonies, reflecting the expression levels of the eight genes, after nine days of continuous feeding (filled symbols) or seven days continuous feeding and two days of starvation (open symbols). The food was either supplemented with LB medium (circles), S. marcescens (squares), E. coli (diamonds), or P. entomophila (triangles). The results for the control Exposure are repeatedly shown for each bacteria Exposure for clarity. Error bars show $95 \%$ confidence intervals for the mean PC1-score across colonies. Letters indicate significant differences within each Exposure and are not directly comparable between exposures. 


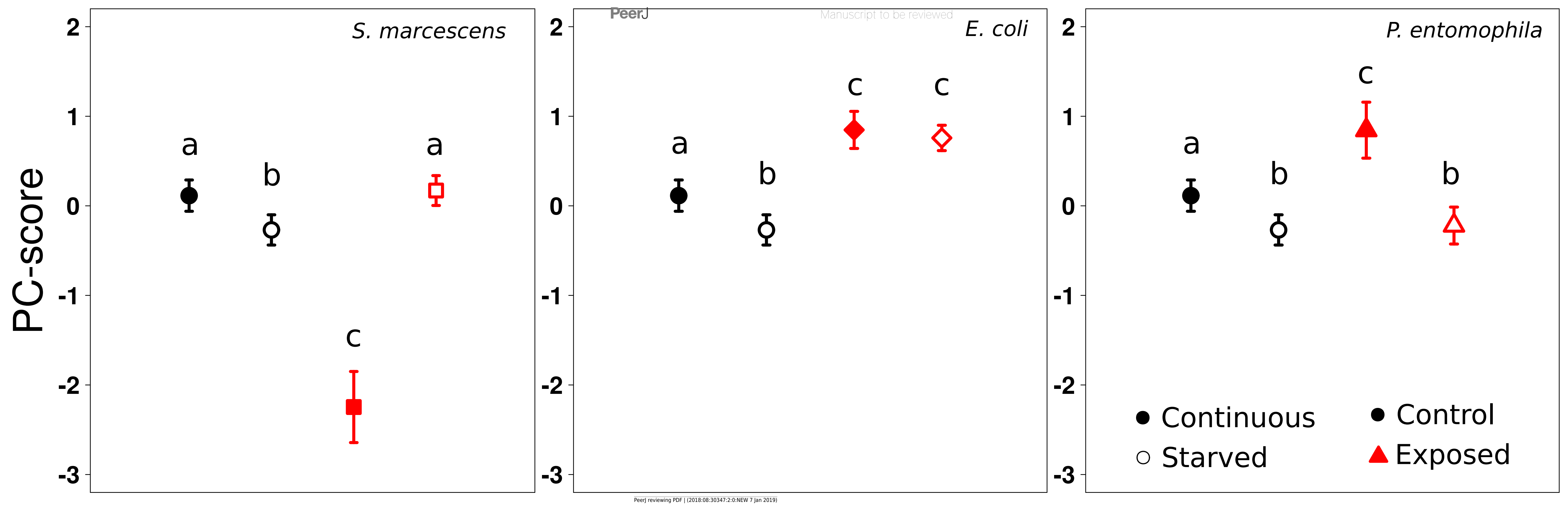




\section{Table $\mathbf{1}$ (on next page)}

Effect of treatments on ant survival

Results from the ad hoc Cox proportional-hazard regression on the influence of each factor (and interactions) on the survival of ants. The hazard rate coefficient $(\beta)$ indicates the change in the probability to die from the treatment/factor compared to the corresponding control treatment/factor. Starvation indicates the comparison of starved to continuously exposed conditions, and the bacteria names correspond to the effect of supplementing the food with S. marcescens, P. entomophila or E. coli, as compared to the control conditions. 
1 Table 1: Effect of treatments on ant survival

2 Results from the ad hoc Cox proportional-hazard regression on the influence of each factor (and

3 interactions) on the survival of ants. The hazard rate coefficient $(\beta)$ indicates the change in the

4 probability to die from the treatment/factor compared to the corresponding control

5 treatment/factor. Starvation indicates the comparison of starved to continuously exposed

6 conditions, and the bacteria names correspond to the effect of supplementing the food with $S$.

7 marcescens, P. entomophila or E. coli, as compared to the control conditions.

\begin{tabular}{llll}
\hline & $\beta \pm \mathrm{SE}$ & $\mathrm{z}$ & $\mathrm{p}$ \\
\hline Starved & $2.46 \pm 0.20$ & 12.26 & $<0.0001$ \\
\hline E. coli & $0.11 \pm 0.19$ & 0.57 & 0.57 \\
\hline S. marcescens & $0.73 \pm 0.17$ & 4.31 & $<0.0001$ \\
\hline P. entomophila & $0.21 \pm 0.18$ & 1.16 & 0.25 \\
\hline Starved*E. coli & $-0.51 \pm 0.24$ & -2.11 & 0.035 \\
\hline Starved*S. marcescens & $-0.36 \pm 0.23$ & -1.58 & 0.11 \\
\hline Starved*P. entomophila & $-0.61 \pm 0.24$ & -2.51 & 0.012 \\
\hline
\end{tabular}

8 


\section{Table 2 (on next page)}

Pairwise comparison of ant survival

Results from the planned post hoc pairwise comparisons of survival between Treatments (Continuously exposed, Starved) within each Exposure regime, as well as between each bacterial Exposure regime (Control exposure, S. marcescens, E. coli, P. entomophila) and the control exposure within each Treatment. All p-values were adjusted for multiple coparisons using false discovery rates. 
1 Table 2: Pairwise comparison of ant survival

2 Results from the planned post hoc pairwise comparisons of survival between Treatments

3 (Continuously exposed, Starved) within each Exposure regime, as well as between each bacterial

4 Exposure regime (Control exposure, S. marcescens, E. coli, P. entomophila) and the control

5 exposure within each Treatment. All p-values were adjusted for multiple coparisons using false

6 discovery rates.

\begin{tabular}{llll}
\hline Exposure/Treatment & $\beta \pm \mathrm{SE}$ & $\mathrm{z}$ & $\mathrm{p}$ \\
\hline Control/Continuous vs Control/Starved & $-2.46 \pm 0.20$ & -12.26 & $<0.0001$ \\
& & & \\
\hline S. marcescens/Continuous vs S. marcescens/Starved & $-1.95 \pm 0.20$ & -9.58 & $<0.0001$
\end{tabular}

E. coli/Continuous vs $E$. coli/Starved

$-2.10 \pm 0.18-11.47<0.0001$

P. entomophila/Continuous vs $P$. entomophila/Starved $\quad-1.85 \pm 0.20 \quad-9.17 \quad<0.0001$

S. marcescens/Continuous vs Control/Continuous $\quad-0.73 \pm 0.17 \quad-4.31<0.0001$

S. marcescens/Starved vs Control/Starved $\quad-0.37 \pm 0.15 \quad-2.50 \quad 0.0168$

E. coli/Continuous vs Control/Continuous $\quad \begin{array}{lll}-0.11 \pm 0.19 & -0.58 & 0.60\end{array}$

\begin{tabular}{llll}
\hline E. coli/Starved vs Control/Starved & $0.41 \pm 0.16$ & 2.62 & 0.0141
\end{tabular}

$\begin{array}{llll}\text { P. entomophila/Continuous vs Control/Continuous } \quad-0.21 \pm 0.18 & -1.16 & 0.31\end{array}$

$\begin{array}{llll}\text { P. entomophila/Starved vs Control/Starved } & 0.39 \pm 0.16 & 2.50 & 0.0168\end{array}$ 


\section{Table 3(on next page)}

Effect of treatments on gene expression

Results from an ad hoc linear mixed effects model on the effect of Exposure (Control, $S$.

marcescens, E. coli, P. entomophila) and Treatment (Continuously exposed, Starved) on the scores of the selected Principal Component (PC1) reflecting gene expression. Parameter estimates are given as $\beta$ plus/minus standard error (SE). 
1 Table 3: Effect of treatments on gene expression

2 Results from an ad hoc linear mixed effects model on the effect of Exposure (Control, $S$.

3 marcescens, E. coli, P. entomophila) and Treatment (Continuously exposed, Starved) on the

4 scores of the selected Principal Component (PC1) reflecting gene expression. Parameter

5 estimates are given as $\beta$ plus/minus standard error (SE).

\begin{tabular}{llll}
\hline & $\beta \pm \mathrm{SE}$ & $\mathrm{t}$ & $\mathrm{p}$ \\
\hline Starved & $-0.38 \pm 0.15$ & -2.64 & 0.0104 \\
\hline E. coli & $0.73 \pm 0.15$ & 5.06 & $<0.0001$ \\
\hline S. marcescens & $-2.36 \pm 0.15$ & -16.27 & $<0.0001$ \\
\hline P. entomophila & $0.73 \pm 0.15$ & 5.04 & $<0.0001$ \\
\hline Starved*E. coli & $0.29 \pm 0.21$ & 1.43 & 0.16 \\
\hline Starved*S. marcescens & $2.80 \pm 0.21$ & 13.65 & $<0.0001$ \\
\hline Starved*P. entomophila & $-0.68 \pm 0.21$ & -3.33 & 0.0015 \\
\hline
\end{tabular}

6 


\section{Table 4 (on next page)}

Pairwise comparison of gene expression

Results from the planned post hoc pairwise comparisons of gene expression reflected as the scores from the selected component (PC1) of a PCA. Comparisons were performed between Treatments (Continuously exposed, Starved) within each Exposure regime, as well as between each bacterial Exposure regime (S. marcescens, E. coli, P. entomophila) and the control exposure within each Treatment. All p-values were adjusted for multiple comparisons using false discovery rates. 
1 Table 4: Pairwise comparison of gene expression

2 Results from the planned post hoc pairwise comparisons of gene expression reflected as the

3 scores from the selected component (PC1) of a PCA. Comparisons were performed between

4 Treatments (Continuously exposed, Starved) within each Exposure regime, as well as between

5 each bacterial Exposure regime (S. marcescens, E. coli, P. entomophila) and the control exposure

6 within each Treatment. All p-values were adjusted for multiple comparisons using false

7 discovery rates.

\begin{tabular}{|c|c|c|c|}
\hline Exposure/Treatment & $\beta \pm \mathrm{SE}$ & $\mathrm{z}$ & $\mathrm{p}$ \\
\hline Control/Continuous vs Control/Starved & $-0.38 \pm 0.15$ & 2.64 & 0.0102 \\
\hline S. marcescens/Continuous vs S. marcescens/Starved & $2.42 \pm 0.15$ & -16.66 & $<0.0001$ \\
\hline E. coli/Continuous vs E. coli/Starved & $-0.09 \pm 0.15$ & 0.62 & 0.61 \\
\hline P. entomophila/Continuous vs $P$. entomophila/Starved & $-1.07 \pm 0.15$ & 7.34 & $<0.0001$ \\
\hline S. marcescens/Continuous vs Control/Continuous & $-2.36 \pm 0.15$ & 16.27 & $<0.0001$ \\
\hline S. marcescens/Starved vs Control/Starved & $0.44 \pm 0.15$ & -3.03 & 0.0036 \\
\hline E. coli/Continuous vs Control/Continuous & $0.73 \pm 0.15$ & -5.06 & $<0.0001$ \\
\hline E. coli/Starved vs Control/Starved & $1.03 \pm 0.15$ & -7.08 & $<0.0001$ \\
\hline P. entomophila/Continuous vs Control/Continuous & $0.73 \pm 0.15$ & -5.04 & $<0.0001$ \\
\hline P. entomophila/Starved vs Control/Starved & $0.05 \pm 0.15$ & -0.34 & 0.79 \\
\hline
\end{tabular}

